\title{
一个渔业模型分年龄最佳收获的探讨 $(\mathbf{I})$ \\ 一一模 型 和 结 论*
}

\author{
刘来福刘继红 \\ (北京师范大学数学系, 北京 100875)
}

关词分年龄收获、最佳渔获、渔获策略

可再生资源的持续收获是资源管理的一个重要课题. 生物种群作为可再生资源, 其年龄 结构在收获过程中是不容忽略的因素, 在分年龄收获下生物种群最大可持续产量是制定分年 龄收获策略的重要参考指标。有关这方面的研究自 1973 年以来已陆续展开. 早期的工作都 是研究线性种群模型的分年砱收获 ${ }^{[1,2]}$. 考虑到线性模型的局限性, 近年来已试图将分年龄收 获用于具有密度制约(即非线性)的种群模型上去 ${ }^{[3-5]}$. 他们把问题归结为线性规划和单变量 函数最大值的问题. 结论是最大可持续产量将在“两年龄组收获”策略下达到, 即该产量将最 多由收获两个年龄组而得到: 只收获某一个年龄组（部分或全部）或者部分收获一个年龄组 (第 $i$ 组)而全部收获另一个年龄组(第 $i$ 组, $i<i$ ), 但具体收获策略将由线性规划给出.

本文将具体讨论一类渔业模型的最佳渔获问题. 模型中密度制约作用将出现在生育(或 鱼卵鄀化)过程中. 这个模型可以用来描述条纹鲇鱼、鳕鱼及鳀鱼等鱼类种群的动态. 在文献 [3]的基础上,本文解析地讨论了此类模型在分年龄收获下最大可持续产量方面的问题. 较之 线性规划, 所得的结果更加直观并为渔获策略的制定提供更多的信息.

设 $\left.N(t)-\left(n_{1}(t)\right), \cdots, n_{m}(t)\right)^{T}$ 为 $t$ 时刻的种群向量, $n_{j}(t)$ 为第 $j$ 年龄组的种群数. 考 虔如下鱼类种群的矩阵模型 ${ }^{[3]}$

$$
\boldsymbol{N}(t+1)=M(\boldsymbol{N}(t)) \boldsymbol{N}(t),
$$

$\boldsymbol{M}(\boldsymbol{N}(\boldsymbol{t}))$ 为带有密度制约的射影矩阵,

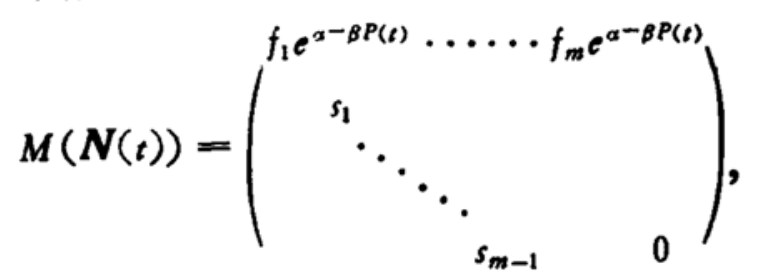

其中 $s_{i}$ 为第 $i$ 年龄组的存活率, $f_{i}$ 为第 $i$ 年龄组的相对繁殖力, $P(t)=e^{a} \sum_{i=1}^{m} f_{i} n_{i}(t)$ 为时间

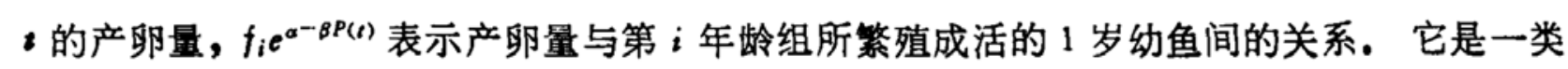
Logistic 矩阵模型 ${ }^{[6]}$.

用 $d_{i}\left(0 \leqslant d_{i} \leqslant 1\right)$ 表示在分年龄捕捞下第 $i$ 年龄组的逃逸率，捕捞率为 $h_{i}=1-d_{i}$.

本文 1989 年 6 月 21 日收到. 1990 年 2 月 26 日收到冬改稿.

* 国家自然科学基金资助项目. 
令 $D=\operatorname{diag}\left(d_{1}, \cdots, d_{m}\right), H=\operatorname{diag}\left(h_{1}, \cdots, h_{m}\right)$, 则在分年龄捕捞下的种群法态将由如下 模型描述:

$$
N(t+1)=D M(N(t)) N(t) \text {. }
$$

产量为 $Y(t)=\boldsymbol{w}^{T} H M(\boldsymbol{N}(t)) \boldsymbol{N}(t)$, 这里 $\boldsymbol{w}=\left(w_{1}, \cdots, w_{m}\right)^{T}$ 是分年龄捕摸的权重向量. $\boldsymbol{w}_{i}$ 可理解为第 $i$ 年龄组个体的平均生物量或平均价值, 这时 $Y(t)$ 将表示渔获的总生物产量或 总产值. 为确定起见, 本文将把 $\boldsymbol{w}$ 理解为平均生物量权重.

模型 (1)的可持续产量将在其平衡解 $\boldsymbol{N}^{*}$ 处达到. (1) 式的平衡解存在、唯一且有形式

$$
v_{i}^{*}=\frac{\ln R_{D}}{\beta R_{D}} D_{i} s_{i-1}, j=1, \cdots, m,
$$

其中 $D_{i}-\prod_{k=1}^{j} d_{k}, S_{0}=1, S_{i}=\prod_{k=1}^{j} S_{k}, R_{D}-e^{\alpha} \sum_{k=1}^{m} D_{k} S_{k} f_{k}, R_{D}$ 表示当 $\beta=0$ (非密度制 约)时捕捞种群(1)式的净生育力. 设非捕捞种群是增长型的, 即其净生育力 $R-R_{D} \mid D=1>$ 1.

由文献 [ 3], 我们将在两年龄组捕捞的情况下讨论最大可持续产量 $Y_{s}$ 的问题. 设第 $i, i$ 组 $(i<i)$, 可能被捕捞, 则在模型 (1) 中将有 $0 \leqslant d_{i}, d_{i} \leqslant 1, d_{k}-1, k \neq i, i$. 我们称之 为捕捞策略 $\left(d_{i}, d_{i}\right)$.

记 $R_{i}=e^{\alpha} \sum_{k=1}^{j} S_{k-1} f_{k}$, 它给出了当 $\beta=0$ 时非捕捞种群从出生一直到第 $i$ 年龄组的净生 育力. 显然 $R=R_{m} . R_{i j}-R_{j}-R_{i-1}(i \leqslant i)$ 给出了第 $j$ 年龄组到第 $j$ 组的净生育力. 在 策略 $\left(d_{i}, d_{i}\right)$ 下, 有

$$
R_{D}=R_{i-1}+d_{i}\left(R_{i j-1}+d_{i} R_{i m}\right)
$$

种群(1)式的可持续产量为

$$
Y_{B}\left(d_{i}, d_{i}\right)=\frac{\ln R_{D}}{\beta R_{D}}\left[w_{i}\left(1-d_{i}\right) S_{i-1}+w_{i} d_{i}\left(1-d_{i}\right) S_{i-1}\right] .
$$

显然, $R_{D}>1$ 是保持有正持续产量的充要条件. 即在策略 $\left(d_{i}, d_{i}\right)$ 下当且仅当 $\beta-0$ 的捕 措种群保持增长的趋势时方可维持正的持续产量. $R_{D} \leqslant 1$ 意味着策略 $\left(d_{i}, d_{i}\right)$ 将导致捕捞 种群旺下降而最终至灭绝的趋势, 即出现 “过度捕拹”的现象. 因此 $R_{D} \leqslant 1$ 就是过度捕捞的 充要条件. 由 $(2)$ 式知, 正方形 $0 \leqslant d_{i}, d_{j} \leqslant 1$ 内满足条件

$$
d_{i} \leqslant\left(1-R_{i-1}\right) /\left(R_{i j-1}+d_{i} R_{j m}\right)
$$

菂子集 $E_{1}$ 将使 $R_{D} \leqslant 1$, 故 $E_{1}$ 为策略 $\left(d_{i}, d_{i}\right)$ 的过度捕捞域,而其余集 $E_{2}$ 为合理捕捞域.

策略 $\left(d_{i}, d_{i}\right)$ 下的最大可持续产量是指限制 $\left(d_{i}, d_{i}\right) \in E_{2}$ 下可持续产量 $Y_{E}\left(d_{i}, d_{i}\right)$ 的 极大值, 即

$$
Y_{s}=Y_{E}\left(d_{i}^{*}, d_{i}^{*}\right)=\max _{\left(d_{i}, d_{i}\right) \in B_{l}} Y_{E}\left(d_{i}, d_{i}\right)
$$

由(2)式, $Y_{E}$ 可写成 $R_{D}, d_{i}$ 的函数

$$
Y_{E}\left(R_{D}, d_{i}\right)=\frac{\ln R_{D}}{\beta R_{D}}\left[A-G\left(d_{i}\right)\left(R_{D}-R_{i-1}\right)\right],
$$

其中 $G\left(d_{i}\right)-\left(B+d_{i} C\right) /\left(R_{i j-1}+d_{i} R_{i m}\right), A=w_{i} S_{i-1}, C=w_{i} S_{i-1}, B=A-C$. 限制域 $\boldsymbol{E}_{2}$ 变为,

$$
B_{3}: 0 \leqslant d_{i} \leqslant 1, r_{0}=\max \left(R_{i-1}, 1\right)<R_{D} \leqslant \max \left(1, r_{1}\left(d_{i}\right)\right),
$$


其中 $r_{1}\left(d_{i}\right)-R_{D}\left(1, d_{i}\right)-R_{i-1}+d_{i} R_{i m}$.

给定 $d_{i}$, 为极大化 $y_{E}\left(R_{D}, d_{i}\right)$, 令

$$
\frac{\partial Y_{E}}{\partial R_{D}}=\frac{1}{\beta R_{D}^{2}}\left[A-R_{i-1} G\left(d_{i}\right)\right]\left[1-\ln R_{D}-A\left(d_{i}\right) R_{D}\right]=0,
$$

其中 $A\left(d_{i}\right)-G\left(d_{i}\right) /\left[A+R_{i-1} G\left(d_{i}\right)\right]=\left[B+C d_{i}\right] /\left[K+L d_{i}\right], K=A R_{i-1}-C R_{i-1}, L=$ $A R-K$, 则方程(5)等价于

$$
1-A\left(d_{i}\right) R_{D}=\ln R_{D} .
$$

可证, 方程 $1-A_{0} R_{D}-\ln R_{D}$ 在区间 $\left[-c^{2},+\infty\right)$ 上定义了单调减的隐函数 $R_{D}\left(A_{0}\right)$, 它在 $\left(0, c^{2}\right]$ 上取值. 当 $K C-B L>0$ 时函数 $A\left(d_{i}\right)$ 单调增, 而 $K C-B L<0$ 时单调㺂. 当 $d_{i}-1$ 时有 $A(1)=R^{-1}>0$.

在我们的问题中, 存在 $0 \leqslant d_{i}<1$, 使得方程 (6) 在 $\left[d_{i}, 1\right]$ 内可解. 给定 $d_{i} \in\left[d_{i}, 1\right]$, (6)式的解 $R_{D}^{*}\left(d_{j}\right) \in\left(0, e^{2}\right]$ 将是 $Y_{E}\left(R_{D}, d_{j}\right)$ 关于 $R_{D}$ 的极大点. 称 $R_{D}^{*}\left(d_{j}\right)$ 为策略 $\left(\cdot, d_{i}\right)$ 下种群的最优净生育力. 它表示为得到最佳捕捞量所必须的净生育力. 最大可持续产量将在 $\left\{R_{D}^{*}\left(d_{i}\right)\right\}$ 中达到.

对于最优净生育力,由(4)式可得持续产量

$$
Y_{B}\left(R_{D}^{*}, d_{i}\right)=\frac{A}{\beta} \frac{\left(\ln R_{D}^{*}\right)^{2}}{R_{D}^{*}-R_{i-1}\left(1-\ln R_{D}^{*}\right)},
$$

它在 $\left[r_{0}, e^{2}\right]$ 内单调增. 于是最大可持续捕拹策略基本上就相当于在限制 $\left(d_{i}, R_{D}^{*}\right) \in E_{3}$ 下 极大化由(6)式所给出的隐函数 $R_{D}^{*}\left(d_{i}\right)$ 的问题. 记 $R_{D}^{*}(0), R_{D}^{*}(1)$ 分别为 $d_{i}=0,1$ 时方 程(6)的解.

由于 $K C-B L$ 的符号决定了 $A\left(d_{i}\right)$ 和 $R_{D}^{*}\left(d_{i}\right)$ 的增减性. 因此它是确定最佳捕拹策 略的一个重要指标. 设 $I_{i}-w_{j} S_{i} / R_{i m}$, 可以证明 $I_{j}-I_{i}$ 将与 $K C-B L$ 同号, 其生物学 含意将另文给出.

如果 $A(0)<-e^{-2}, R_{D}^{*}(0)$ 将不存在. 由于 $A(1)=R^{-1}>0, A\left(d_{i}\right)$ 单调增. 令 $d_{i}=-\left(B+K e^{-2}\right) /\left(C+L e^{-2}\right)$ 则 $0<d_{i}<1$, 它是方程 $A\left(d_{i}\right)=-e^{-2}$ 的解. $R_{D}^{*}\left(d_{i}\right)$ 的条件极大将在 $\left[\tilde{d}_{i}, 1\right]$ 内寻求.

$I_{j}-I_{i}>0$ 时如果 $R_{D}^{*}\left(d_{i}\right)$ 与限制域 $E_{3}$ 的上缘 $\max \left(1, r_{1}\left(d_{i}\right)\right)$ 有交点, 则交点处的 $R_{D}^{*}$ 即所求的极值, 它是方程

$$
1-A\left(d_{j}\left(R_{D}\right)\right) R_{D}=\ln R_{D}
$$

\begin{tabular}{|c|c|c|c|c|c|}
\hline & \multicolumn{4}{|c|}{$I_{i}<I_{j}$} & \multirow{3}{*}{$\underbrace{I_{i}>I_{i}}_{r_{0}<R_{D}^{*}(1) \leqslant R_{i-1}}$} \\
\hline & \multicolumn{2}{|c|}{$A(0)<-e^{-2}$} & \multicolumn{2}{|c|}{$A(0) \geqslant-e^{-2}$} & \\
\hline & $R_{\delta}^{*}\left(d_{i}\right) \leqslant r_{1}\left(d_{i}\right)$ & $1<r_{1}\left(d_{i}\right)<R_{D}^{*}\left(d_{i}\right)$ & $r_{0}<R_{D}^{*}(0) \leqslant R_{i-1}$ & $1<R_{j-1}<R_{D}^{*}(0)$ & \\
\hline$d_{i}$ & $\frac{R_{D}^{*}-R_{i-1}}{R_{i-1}+d_{i} R_{i m}}$ & & $\frac{R_{D}^{*}-R_{i-1}}{R_{i i-1}}$ & & $\frac{R_{l}^{*} !}{R_{i m}}=R_{i-1}$ \\
\hline$d^{*}$ & $d_{i}$ & $\frac{R_{D}^{*}-R_{i-1}}{R_{i}}$ & 0 & $\frac{R_{D}^{*}-R_{i-1}}{R_{i}}$ & 1 \\
\hline $\boldsymbol{R}_{\text {弗 }}$ & $R_{D}^{*}\left(d_{i}\right)=e^{2}$ & $R_{D}^{*}$ & & $R_{D}^{*}$ & $R_{D}^{*}(1)$ \\
\hline
\end{tabular}

满足条件 $R_{D}>1$ 的解, 这里 $d_{i}\left(R_{D}\right)=\left(R_{D}-R_{i-1}\right) / R_{i m}$.

表 1 最大可持续捕捞策略 $\left(d_{i}^{*}, d_{i}^{*}\right)$ 及其最优净生育力 $R_{D}^{*}$ 
通过对各种情况下函数 $R_{D}^{*}\left(d_{i}\right)$ 条件极大值的讨论可得收获第 $i, i$ 组时表 1 所列的最大 可持续捕捞策略 $\left(d_{i}^{*}, d_{i}^{*}\right)$ 及相应的最优净生育力 $R_{D}^{*}$, 最大可持续产量可由 (3) 式或 (4) 式 给出. 表中第三、五列的策略为只部分收获第 $i$ 组当 $r_{1}\left(d_{i}\right)>1$ 或 $R_{i-1}>1$ 不满足时, 如 果(7)式有解 $R_{D}^{*}>1$, 结论仍然成立. 否则将不存在持续捕捞策略. 第四列为部分收获第 $i$ 组而全部收获第 $i$ 组, 第六列为只收获第 $i$ 组. 当条件 $R_{D}^{*}\left(\nu^{0}\right)>r_{0}$ 或 $R_{D}^{*}(1)>r_{0}$ 不满贫 时, 持续捕拹仅当 $R_{D}^{*}(0)$ 或 $R_{D}^{*}(1)$ 在 $\left(1, R_{i-1}\right)$ 内时发生. 这时将全部捕拹第 $i$ 组 $d_{i}^{*}=$ $0, R_{D}^{*}=R_{i}$, 而无 $d_{i}^{*}$ 可言. 第一列为部分收获第 $i, i$ 两个年龄组.

通过对不同年龄组最佳捕拹策略的比较分析, 就可以得到最佳捕捞的年龄组及其最大可 持续捕捞策略.

结论的生物学解释及在渔业捕捞上的数值例子将在以后给出.

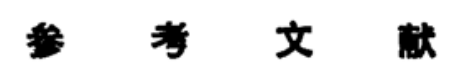

[ 1 ] Beddington, J. R. and Taylor, D. B., Biometrics, 29 (1973), 801-809.

[2] Rorres, C., Biometrics, 32(1976), 945-948.

[ 3 ] Reed, W. T., Biometrics, 36(1980), 579--693.

[ 4 ] Getz, W. M., Math. Bioscience, 48(1980), 279-292.

[ 5 ] Getz, W. M. and Haight, R. G., Population Harvesting: Demographic Models of Fish, Forest and Animal Resources, Princeton University Press, New Jersey, 1989.

[6] Liu, L. and Cohen, J. E., J. Math. Biol., 25(1987), 73-88. 